\title{
Scaling Equations for Ballistic Modeling of Solid Rocket Motor Case Breach
}

\author{
Joshua E. McMillin* \\ ATK Launch Systems, Brigham City, UT, 84302-0707
}

\begin{abstract}
This paper explores the development of a series of scaling equations that can take a known nominal motor performance and scale it for small and growing case failures. This model was developed for the Malfunction-Turn Study as part of Return to Flight activities for the Space Shuttle program. To verify the model, data from the Challenger accident (STS$51 \mathrm{~L}$ ) were used. The model is able to predict the motor performance beyond the last recorded Challenger data and show how the failed right hand booster would have performed if the vehicle had remained intact.
\end{abstract}

\section{Nomenclature}

$\gamma \quad=$ Ratio of the specific heats for a gas

$\rho_{\text {prop }} \quad=$ Propellant density

$\rho_{g} \quad=$ Combustion gas density

$45 \mathrm{SW}=45^{\text {th }}$ Space Wing - Patrick Air Force Base

$a \quad=$ Propellant burn rate constant

$A^{*} \quad=$ Nozzle throat area

$A_{B} \quad=$ Area of case breach

$A_{e} \quad=\quad$ Nozzle exit area

$A_{S} \quad=$ Total propellant surface area

$A^{t} \quad=$ Total effective throat area

$C^{*} \quad=$ Effective exhaust velocity

$C_{\text {Ref }}^{*}=C^{*}$ at reference pressure

$C_{F} \quad=$ Thrust coefficient

$D \quad=$ Total burn distance

$F \quad=$ Thrust

$F_{B} \quad=$ Anomalous thrust due to case breach

$i=$ Data index used when scaling nominal performance

$M \quad=$ Mach number or total expended mass

$\dot{m} \quad=$ Mass flow rate

$n=$ Burn rate pressure exponent

$P \quad=$ Pressure

$P_{a} \quad=$ Ambient pressure

$P_{c} \quad=$ Chamber stagnation pressure

$P_{e} \quad=\quad$ Nozzle exit plane static pressure

$P_{\text {Ref }} \quad=$ Reference pressure

$q=\mathrm{C}^{*}$ sensitivity to pressure

$\dot{r} \quad=$ Propellant burn rate

RSRM $=$ Reusable Solid Rocket Motor

STS $=$ Space Transportation System

$T=$ Time

$\Delta t \quad=$ Time interval

$X_{\text {nom }} \quad=$ Value of parameter $X$ for nominal motor

$X_{\mathrm{dev}}=$ Value of parameter $X$ for deviating motor

\footnotetext{
"Mechanical Engineer, RSRM Ballistic \& Grain Design Team, M/S LD1, Member.
} 


\section{Introduction}

$\mathrm{T}$ HIS paper explores the development of a series of scaling equations that can scale known nominal motor performance for small and growing case failures that result in overboard combustion gas venting but not immediate case rupture. Previous modeling efforts assumed a simple 2-12 second decay to zero thrust from the time of failure. In June of 2003, the RSRM ballistics team was asked to revisit these analyses to support the updated Launch Area Risk Assessment performed by the 45th Space Wing (45SW) and ACTA, Inc. It was determined that a more realistic, or physics based, failure model was needed. The resulting failure model simulated a growing hole in the case wall. The model parametrically scales motor performance for a given time of failure initiation. The failure affects time, chamber pressure, thrust, and mass flow rates. This model was provided to NASA's Johnson Space Center and incorporated into the $1^{\text {st }}$ Stage Malfunction-Turn Study.

The evaluation of these failure models was purposefully initiated for conducting contingency analyses. They do not reflect a true shortcoming of the motor design. Failures such as these are extremely unlikely due to the robustness of the motor case and joint seal design.

\section{Background}

The 1st Stage Malfunction-Turn Study provided nominal and malfunction turn trajectories in support of the updated Launch Area Risk Assessment performed by the 45SW and ACTA, Inc. The trajectories were used as inputs for the calculation of casualty expectation values, which the range safety community used to evaluate public risk. Prior to this study, malfunction-turn data had not been provided to the 45SW since STS-5. The delivery of the updated malfunction-turn trajectories was coordinated by the Space Shuttle Range Safety Panel.

The previous (STS-5) Malfunction-Turn Study incorporated two types of RSRM failures modes; single actuator failures and thrust decay failures. The thrust decay methodology employed in that study involved linear or exponential thrust-decay profiles where the thrust decayed to zero over a 2-12 second time interval. Due to the unrealistic nature of these previous models, NASA requested that models be developed that are both more realistic and physics based. The new thrust decay models developed by ATK Launch Systems served as inputs to the trajectory simulation used to generate the data delivered to the $45 \mathrm{SW}$.

The original thrust decay model was intended to simulate a failure where the nozzle throat is burned through. Burn through and subsequent ejection of the nozzle are considered unreasonable for the RSRM because the nozzle throat cannot be physically ejected in one piece. For this situation to occur, either the throat insert would have to break apart, or the entire nozzle would need to be destroyed. The safety factors in the throat region are sufficiently high to keep this from occurring. In addition, it is believed that a complete ejection of the nozzle would not extinguish the motor. Therefore, the propellant grain would continue to burn and thrust would not decay to zero.

In an effort to re-evaluate the models used, ATK Launch Systems developed two models for the new study, neither failure scenario is considered likely. The first model simulated a failure of nozzle joint \#1. The second model simulated a growing breach of the pressure chamber.

Although such a failure is highly unlikely, the failure of nozzle joint \#1 was selected as the most realistic and readily modeled failure point of the RSRM nozzle. Because it has less material, the exit cone liner has a lower safety factor on char and erosion than does the throat, making the potential for burn through at least theoretically higher. Nozzle joint \#1 is the location where the aft exit cone attaches to the forward exit cone of the nozzle assembly. Figure 1 shows a cut away schematic of the RSRM nozzle, with the joint locations identified. Ideally, chamber conditions forward of the throat plane are independent of conditions aft of the throat plane, so a failure of joint \#1 would not impact the propellant grain, nor would the motor performance decay to zero thrust. This was modeled simply as an instantaneous change in the expansion ratio of the nozzle. The math for this can be found in most textbooks covering compressible flow through a converging diverging nozzle.

The degradation of thrust resulting from the nozzle joint \#1 failure resulted in a significantly less severe malfunction-turn than the original study. Based on the assumptions of the Malfunction-Turn Study, and barring any secondary failures, the STS would still reach staging with the nozzle joint \#1 failure. Therefore, it was requested that ATK Launch Systems also provide a model that would lead to continuous, more detrimental, thrust decay. For this level of thrust decay to occur, the nozzle throat, nozzle assembly, or the pressure chamber must fail in some way. 


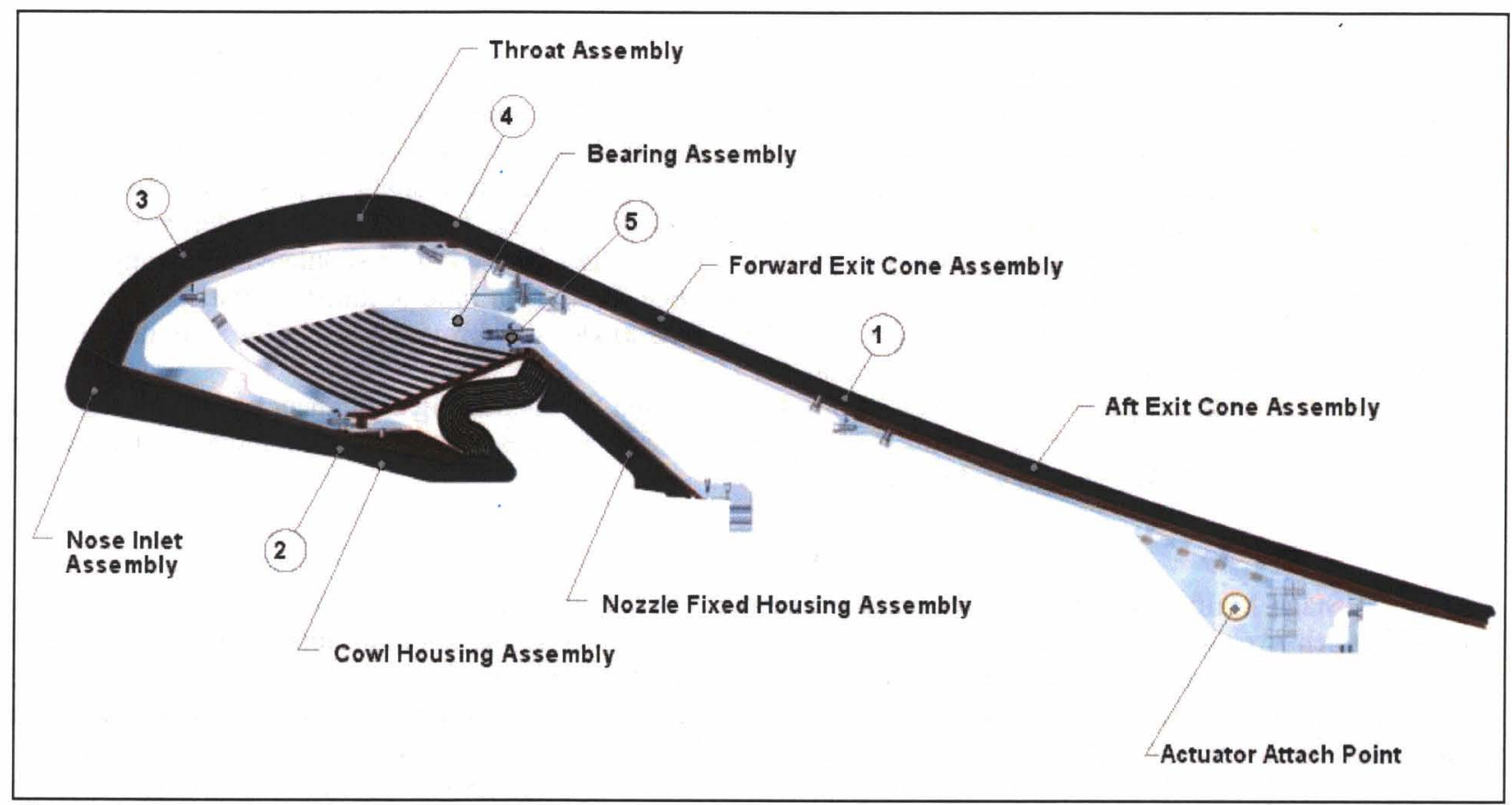

Figure 1: RSRM Nozzle Joint Locations

Failure of the throat through rapid erosion was considered extremely implausible because of the large safety factors and historical performance of the throat ring. Failure of the pressure chamber may occur if a joint seal or the pressure vessel was breached in some other way. The case breach scenario is also highly unlikely; but was chosen because a joint failure actually happened on STS-51L. The failure model is derived such that it is not limited to the joint areas, and could represent a venting breach anywhere in the pressure vessel. Additionally, this same methodology could be used, with some alteration, to model high levels of throat erosion.

Since the Challenger accident, the field joints have been redesigned to prevent an STS-51L type of failure and joint heaters have been added to keep the sealing O-rings at temperatures warm enough to ensure the O-rings track joint movements with large safety margins.

\section{Parametric Model of Case Breach}

\section{A. Scaling Equations}

Modeling the case breach failure is not as straight forward as modeling a failure of nozzle joint \#1. The case breach model is intended to simulate an event in which the pressure chamber is compromised, leading to a thrust decay scenario. Timing, pressure, mass flow, and thrust are all affected due to the nature of this failure. The case breach is modeled as a second nozzle with increasing throat area. The model does not account for axial pressure drop, but rather utilizes a 0-D scaling approach to simulate the failure. This assumption allows the model to be used regardless of where the case breach is located. The equations scale a nominal performance curve for a given failure onset time. Per requirements, the model is able to initiate the failure at any given time. Section B will detail the derivation of the scaling equations for time, pressure, thrust, and mass flow.

Currently the RSRM program uses similar scaling equations for flight predictions. These standard scaling equations adjust a nominal "block motor" performance based on loaded propellant weight, predicted burn rate, and propellant mean bulk temperature. The scaling equations derived for the case breach failure can be used in conjunction with these standard scaling equations.

Figures 2, 3, and 4 show the results of this model applied to a nominal RSRM with a failure initiation time of 25 seconds. The failure is modeled as a circular area with a constant radial growth rate. These results are given as an example only. Final use of the model by engineers at NASA Johnson Space Center used multiple failure initiation times and locations. 


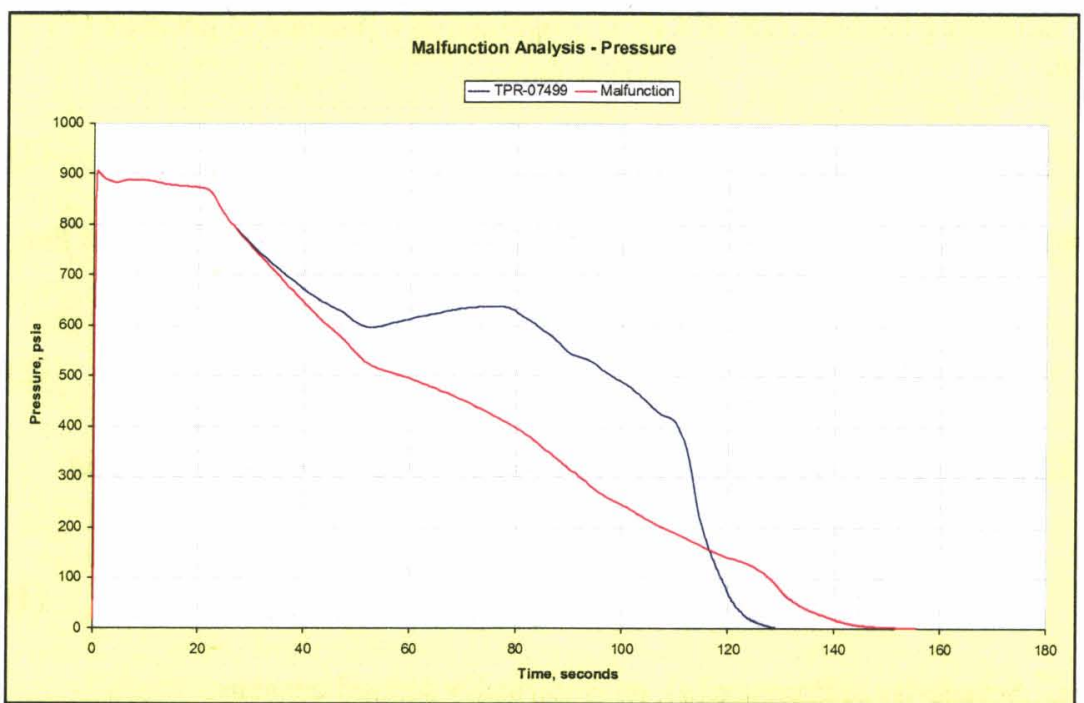

Figure 2: Results From the Parametric Model for Pressure

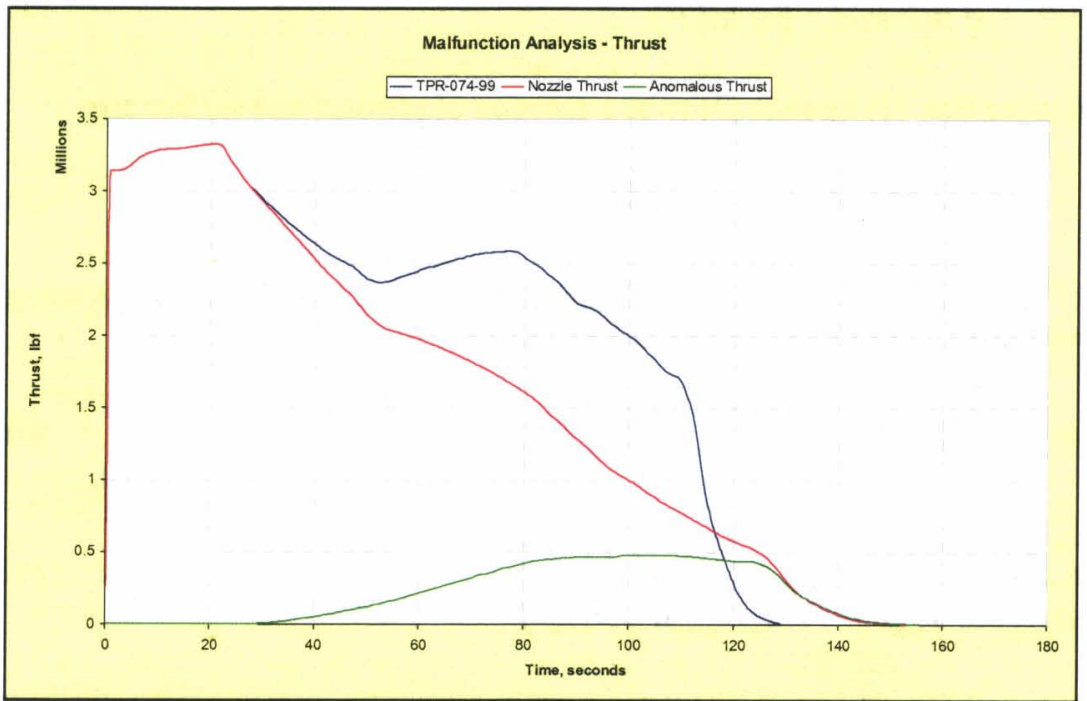

Figure 3: Results From the Parametric Model for Thrust

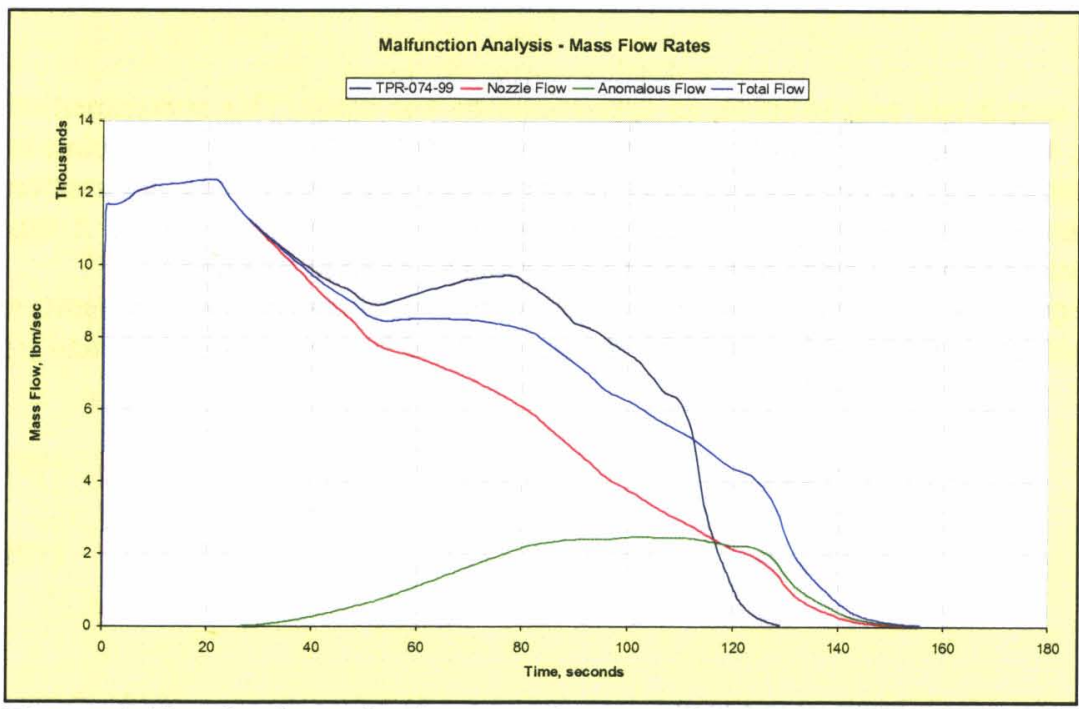

Figure 4: Results from the Parametric model for Mass Flow
B. Deriving the Scaling Equations

A complete list of nomenclature used in the derivation of these equations can be found at the beginning of this paper.

The key assumption in the scaling equation approach is that each data point in the nominal data file represents a fixed burn distance or a fixed propellant geometry. Although the breach is growing, the analysis assumes that steady state equations are still applicable. The result is a set of data correlating time, chamber pressure, thrust for both the nozzle and the breach, mass flow for both the nozzle and the breach, and the breach area.

\section{Pressure Scaling}

Using steady state equations, the nominal head-end pressure can be scaled as a function of the nominal and deviant effective throat areas to predict the deviant pressure trace. The nominal throat area $\left(A^{*}{ }_{n o m}\right)$ is the original area of the nozzle throat associated with a given data point. The deviant throat area $\left(A_{d e v}^{t}\right)$ is the combined total area of the new nozzle throat and case breach. The deviant nozzle throat may be slightly different from the nominal nozzle throat because of normal throat erosion occurring over a longer period.

The steady state assumption means that mass flow being generated at the burning propellant surface is equal to the mass flow being expelled through the nozzle and case breach. There is no appreciable change in the mass stored in the chamber volume. Assuming each data point in the nominal performance represents a fixed burn distance, and therefore a fixed surface area $\left(A_{S}\right)$, the surface area term cancels out along with the propellant density $\left(\rho_{\text {prop }}\right)$ and burn rate constant term $(a)$.

The pressure scaling equation begins with the fundamental equations for Burn rate, Eq. (1), and mass flow, Eq. (2). Ultimately, the result is Eq. (8). 
Equation (1) is a general formula for estimating the burn rate of a solid propellant as a function of pressure $(P)$ and empirically derived constants $a$ and $n$.

$$
\dot{r}=a P^{n}
$$

Equation (2) calculates the mass flow from the propellant surface as a function of surface area the density of the propellant and the burn rate.

$$
\dot{m}=A_{S} \rho_{\text {prop }} \dot{r}
$$

Equation (3) is the definition for the Characteristic Exhaust Velocity $\left(C^{*}\right)$, which relates pressure, total throat area $\left(A^{t}\right)$, and mass flow.

$$
C^{*} \equiv P A^{t} / \dot{m}
$$

Equation (4) is the definition for the $C^{*}$ Sensitivity to Pressure ( $q$ ), an empirically derived constant.

$$
q \equiv \partial \ln \left(C^{*}\right) / d \ln (P)
$$

The equation for $C^{*}$ can be rewritten using Eqs. (1) through (3) to be a function of pressure and surface area.

$$
C^{*}=P^{1-n} A^{t} /\left(A_{S} \rho_{\text {prop }} a\right)
$$

Equation (6) uses Eq. (4) to relate $C^{*}$ at different pressure levels. Equation (7), which comes from substituting Eq. (5) in to Eq. (6), can be solved to give the pressure for the deviant motor, Eq. (8).

$$
\begin{gathered}
C_{\text {Rref }}^{*}=C_{d e v}^{*}\left(P_{\operatorname{Re} f} / P_{d e v}\right)^{q}=C_{\text {nom }}^{*}\left(P_{\operatorname{Re} f} / P_{n o m}\right)^{q} \\
\frac{P_{d e v}^{1-n} A_{d e v}^{t}}{A_{S} \rho_{\text {prop }} a}\left(\frac{P_{\operatorname{Re} f}}{P_{d e v}}\right)^{q}=\frac{P_{n o m}^{1-n} A_{T_{n o m}}^{*}}{A_{S} \rho_{\text {prop }} a}\left(\frac{P_{\operatorname{Re} f}}{P_{n o m}}\right)^{q} \\
P_{d e v}=P_{n o m}\left(A_{T_{n o m}}^{*} / A_{d e v}^{t}\right)^{1 /(1-n-q)}
\end{gathered}
$$

\section{Time Scaling}

To create scaling equations that are simple and easy to solve, an approximation was made. This approximation was that the pressure, nozzle throat area, and breach area remain sufficiently constant between two adjacent points in the nominal performance data. The longer the failure was allowed to grow the more error this assumption introduced. This error was insignificant for sufficiently small increments of time. As seen in section 5 this assumption also affected the mass flow calculations. A more accurate derivation is presented in section 6

The key assumption for the scaling equations is that each data point in the nominal performance data represents a fixed burn distance $(D)$, or a fixed propellant geometry. Therefore, the distance burned between points $[i-1]$ and $[i]$ remains constant as in Eq. (10).

$$
\begin{gathered}
D=\dot{r} \Delta t \\
D_{n o m}=D_{d e v}
\end{gathered}
$$


Combining Eq. (9), Eq. (10), and Eq. (1) relates the nominal pressure and time delta $\left(\Delta t_{\text {nom }}\right)$ with the predicted deviant pressure and time delta $\left(\Delta t_{d e v}\right)$ as seen in Eq. (11).

$$
a P_{n o m}^{n} \Delta t_{n o m}=a P_{d e v}^{n} \Delta t_{d e v}
$$

Substituting Eq. (8) into Eq. (11), the deviant time delta between two data points can be expressed by Eq. (12)

$$
\Delta t_{d e v}=\Delta t_{n o m}\left(A_{T_{\text {nom }}}^{*} / A_{d e v}^{t}\right)^{-n /(1-n-q)}
$$

The time for the deviant performance $\left(T_{d e v, i}\right)$ is the deviant time delta plus the deviant time value for the previous data point $\left(T_{d e v, i-1}\right)$.

$$
T_{d e v, i}=T_{d e v, i-1}+\Delta t_{d e v}
$$

The area of the breach $\left(A_{B}\right)$ can be modeled as any function of time. For the purpose of the Malfunction-Turn Study, this was modeled as a simple circular hole with a fixed growth rate beginning at a given time $\left(T_{B}\right)$.

$$
A_{B}=f\left(T_{d e v}-T_{B}\right)
$$

The total effective throat area $\left(A_{d e v}^{t}\right)$ is the sum of the breach area $\left(A_{B}\right)$ and the nozzle throat area $\left(A^{*}{ }_{T d e v}\right)$.

$$
A_{d e v}^{t}=A_{T_{d e v}}^{*}+A_{B}
$$

Because the breach area increases with increasing time, and because of the slow erosion of the nozzle throat, it is easiest to iterate on Eqs. (12) - (15) until a convergence on time is reached. The spreadsheet provided by ATK Launch Systems to engineers at NASA Johnson Space Center for the Malfunction-Turn Study converges to \pm 0.001 seconds at all times within eight iterations for failure onset at initiation. The bigger the failure is allowed to grow, the more iteration is needed to converge within a given tolerance.

\section{Nozzle Thrust Scaling}

The nozzle thrust $\left(F_{\text {Nozzle }}\right)$ scaling is obtained by assuming that for a given data point in the nominal performance, the thrust coefficient, defined in Eq. (16), remains constant as in Eq. (17). In effect, this also states that the expansion ratio is unaffected. The small change in nozzle expansion ratio is neglected because of the added modeling complexity and because of its relatively small effect.

$$
\begin{gathered}
C_{F} \equiv F_{\text {Nozzle }} / P A^{*} \\
C_{F, \text { dev }}=C_{F, \text { nom }}
\end{gathered}
$$

Substituting Eq. (16) into Eq. (17) results in Eq.(18). This relates thrust to the product of pressure and throat area for both the nominal and deviating conditions.

$$
F_{\text {Nozzle,dev }} /\left(P_{\text {dev }} A_{T_{\text {dev }}}^{*}\right)=F_{\text {Nozzle }, \text { nom }} /\left(P_{\text {nom }} A_{T_{\text {nom }}}^{*}\right)
$$

Equation (19) scales thrust from the nominal performance as a function of effective throat areas using Eq. (8) with Eq. (18).

$$
F_{\text {Nozzle,dev }}=F_{\text {Nozzle }, \text { nom }}\left(A_{T_{\text {dev }}}^{*} / A_{T_{\text {nom }}}^{*}\right)\left(A_{T_{\text {nom }}}^{*} / A_{d e v}^{t}\right)^{1 /(1-q-n)}
$$




\section{Breach Thrust}

The derivation for the breach thrust $\left(F_{B}\right)$ begins with the ideal thrust equation ${ }^{1}$, Eq. (20). This can be greatly simplified given the assumptions of vacuum ambient conditions and choked flow through an orifice, where the throat $\left(A^{*}\right)$ and exit $\left(A_{e}\right)$ areas are both equal to the breach area $\left(A_{B}\right)$.

$$
\begin{gathered}
F=A^{*} P_{c} \gamma\left[\{2 /(\gamma-1)\}\{2 /(\gamma+1)\}^{(\gamma+1) /(\gamma-1)}\left\{1-\left(P_{e} / P_{c}\right)^{(\gamma-1) / \gamma}\right\}\right]^{0.5}+\left(P_{e}-P_{a}\right) A_{e} \\
\mathrm{~A}_{\mathrm{e}}=A^{*} \equiv A_{B}
\end{gathered}
$$

Equation (22) is the isentropic relationship between Mach number and pressure.

$$
P_{e} / P_{c}=\left(1+0.5(\gamma-1) M^{2}\right)^{-\gamma /(\gamma-1)}
$$

The Mach number for choked flow through an orifice is 1.0 and Eqs. (20) - (22) combine to yield Eq. (23).

$$
\left.F_{B}=P_{d e v} A_{B}(\gamma+1)(2 /(\gamma+1))\right)^{/(\gamma-1)}
$$

Adjustments for non-ideal flow losses or dimensional effects are not considered. For the purposes of the Malfunction-Turn Study, the off nominal thrust generated by the breech is kept as high as possible to maximize thrust imbalance between the failed and nominal boosters.

5. Mass Flow Rate Scaling

Mass flow rate scaling is based on conservation of mass, Eq. (24), and assumes that the difference in mass storage is negligible. The mass flowed from data point to data point must be the same in order to meet the assumption that each data point represents a fixed burn distance and fixed propellant geometry. Using a numerical integration technique (trapezoidal rule), Eq. (25), the total mass flow is determined using Eq. (26).

$$
\begin{gathered}
M_{i, d e v}=M_{i, n o m} \\
M_{i}=M_{i-1}+0.5\left(\dot{m}_{i}+\dot{m}_{i-1}\right) \Delta t
\end{gathered}
$$

Equations (24) and (25) combine to form Eq. (26). This is the equation used in the Malfunction-Turn Study to scale the nominal mass flow data for a malfunctioning booster.

$$
\dot{m}_{i, d e v}=\left(\dot{m}_{i, n o m}+\dot{m}_{i-1, n o m}\right)\left(\Delta t_{n o m} / \Delta t_{d e v}\right)-\dot{m}_{i-1, d e v}
$$

Equations (29) and (30) are used to determine the mass flow being ejected from the nozzle and case breach respectively. These were based on the assumption that the same gas species, at identical conditions (constant gas density, $\rho_{g}$ ), are flowing through both the nozzle throat and the breach. Axial pressure drop is ignored in the scaling equations to simplify the approach and allow simulations to use the same equations for a failure at any location. With this assumption, the individual mass flow rates become proportional to the breach and nozzle throat areas.

$$
\begin{gathered}
\dot{m}=\rho_{g} V \cdot A \\
\dot{m} / A^{t}=\dot{m}_{\text {Nozzle }} / A^{*}=\dot{m}_{B} / A_{B} \\
\dot{m}_{\text {Nozzle }}=\dot{m}_{i} A^{*} / A^{t} \\
\dot{m}_{B}=\dot{m}_{i} A_{B} / A^{t}
\end{gathered}
$$




\section{Corrected Mass Flow and Time Scaling}

From Eqs. (1) (2) and (8) listed previously, it is also possible to calculate Eq. (31). Unfortunately, with the time scaling derived in section 2 , this equation will violate the conservation of mass requirement. On an instantaneous basis, this difference is small; however, integrated over time the error became evident. For failure onset at initiation $(\mathrm{T}=0)$ the error accumulated to almost $0.15 \%$ of the RSRM total expended mass.

$$
\dot{m}_{\text {New }}=\dot{m}_{\text {old }} \times\left(A_{T_{\text {old }}}^{*} / A_{\text {New }}^{t}\right)^{n /(1-n-q)}
$$

Upon further investigation, it was found that this error became smaller and smaller with decreasing the magnitude of time increments used in the nominal performance data. Ideally, Eqs. (26) and (31) should yield the same results. The difference has been identified as an inadequacy in Eq. (9), which assumes that the pressure change between data points in the nominal performance is negligible. This assumption breaks down as the failure progresses and the change in the time steps and pressure become larger. It also breaks down for motors having more radical changes in pressure. Equation (32) is a more correct equation for the distance burned between two points.

$$
D=\int \dot{r} d t
$$

Equation (12) can be replaced using Eq. (1), Eq. (10), and a trapezoidal integration rule to approximate Eq. (32)

$$
0.5 a\left(P_{i, d e v}^{n}+P_{i-1, d e v}^{n}\right) \Delta t_{d e v}=0.5 a\left(P_{i, n o m}^{n}+P_{i-1, n o m}^{n}\right) \Delta t_{n o m}
$$

This can be written in terms of the effective throat and known pressures. The new equation is somewhat longer than Eq. (12), but it is easy enough to translate into a computer program or spreadsheet.

$$
\Delta t_{\text {dev }}=\Delta t_{\text {nom }}\left[\begin{array}{l}
\left\{\left(A_{i, \text { nom }}^{*} / A_{i, \text { dev }}^{t}\right)^{n /(1-n-q)}+\left(P_{i-1, \text { dev }} / P_{i, n o m}\right)^{n}\right\}^{-1}+ \\
\left\{\left(A_{i, \text { nom }}^{*} / A_{i, \text { dev }}^{t}\right)^{n /(1-n-q)}\left(P_{i, \text { nom }} / P_{i-1, n o m}\right)^{n}+\left(P_{i-1, \text { dev }} / P_{i-1, n o m}\right)^{n}\right\}^{-1}
\end{array}\right]
$$

Substituting Eq. (34) for Eq. (12) and Eq. (31) for Eq. (30) results in a significantly reduced amount of error in the total integrated mass flow $(<0.0003 \%)$.

\section{Modeling the Case Breach}

For the purpose of the malfunction study, the case breach was modeled as a circular opening in the pressure vessel with a fixed growth rate. There are no known design deficiencies, or preventable scenarios, that could lead to this failure. Modeling of the case breach was performed to provide information used in contingency planning for unforeseeable events. The shape of the failure was selected based on ease of modeling. The growth rate was based on a charring material ablation model of hot propellant combustion products flowing through a circular hole in a flat steel plate. This was considered conservative because it did not include any protective materials such as insulation, liner, or propellant. STS-51L data was also evaluated to confirm the appropriateness of the results. Analysis of the available pressure data indicates that the STS-51L failure grew at a slower rate. Therefore, the model used in the Malfunction-Turn Study was conservative and generated a larger thrust imbalance.

\section{STS-51L}

The STS-51L case breach was initiated by a failure of the aft field joint of the right hand booster. The case breach failure model was used to simulate the performance of that right hand motor. A time of 58.788 seconds was picked as the failure initiation time because it was the time determined by the Challenger accident investigation ${ }^{2}$ for first evidence of flame. The joint failure was modeled in two different ways. First as a circular hole growing with constant radial growth rate. A radial erosion rate of 0.25 -in/sec (Fig. 5) best matched available pressure data with this assumption. A radial erosion rate of 0.32 -in/sec (Fig. 6) was needed to match the final failure area with a constant radial growth. Second, the failure was modeled with a constant area growth rate. With this assumption, a 


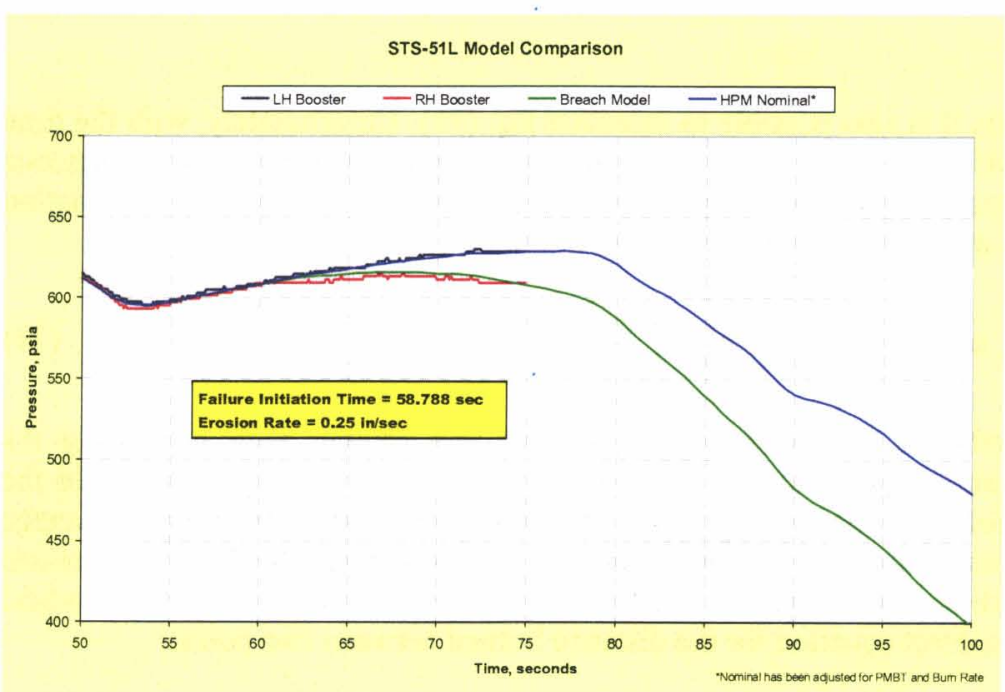

Figure 5: STS-51L Modeled with 0.25 -in/sec Radial Growth Rate

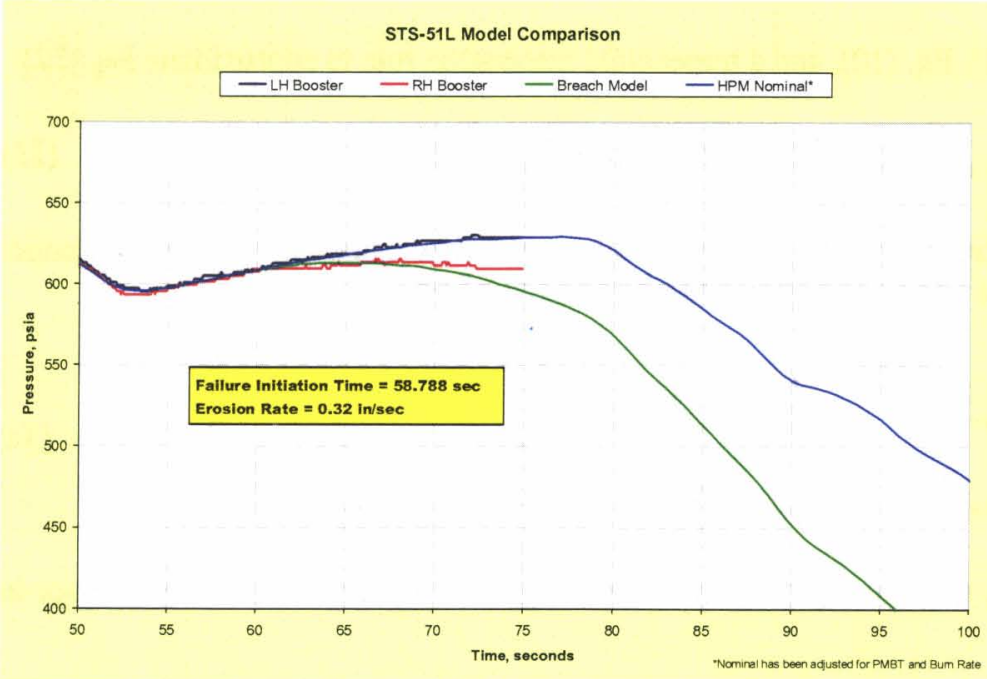

Figure 6: STS-51L Modeled With 0.32-in/sec Radial Growth Rate

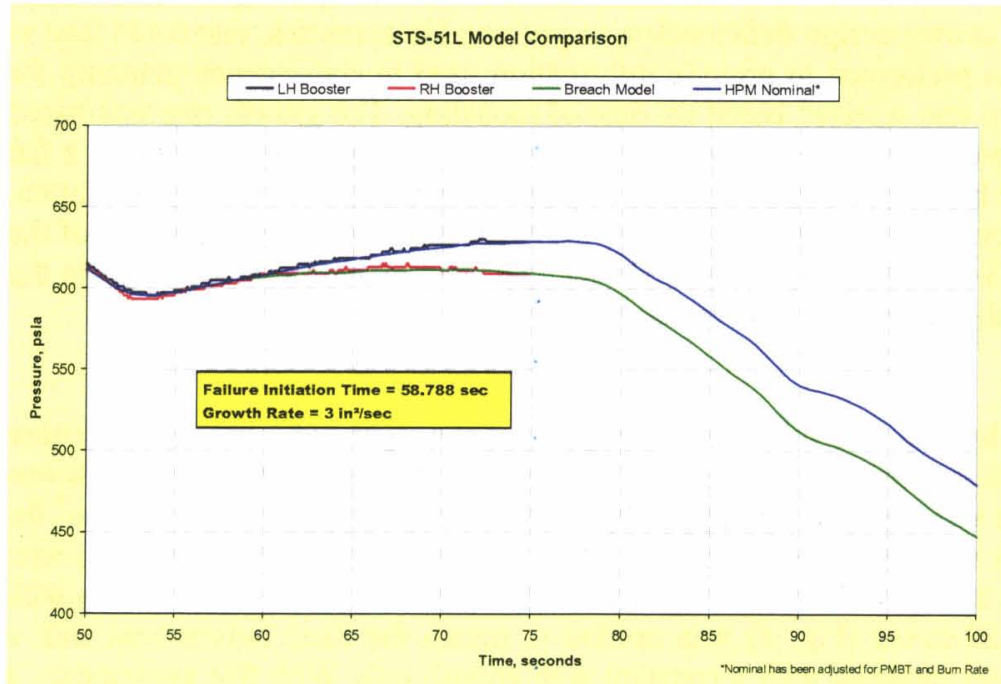

constant area growth rate of $3-\mathrm{in}^{2} / \mathrm{sec}$ best matched the available chamber pressure data. Clearly, the results obtained depend on the assumptions made about the geometry and growth of the venting failure.

The recovery of the STS-51L hardware allowed for an estimate of the final dimensions of the case failure. A bottom portion of the aft cylinder of the aft center segment was recovered with a burned out area approximately 15 axial inches and 28.25 circumferential inches. A portion of the aft attach cylinder was also recovered with evidence of burn through defining the bottom edge of the STS-51L failure. Figure 8 and Fig. 9 show the dimensions of the case segments recovered with evidence of the burn through. From these two pieces, it was possible to estimate the size of the failure at the time of destruction. According to the failure investigation ${ }^{3}$, the failure was approximately $862-\mathrm{in}^{2}$. If the failure can be modeled as a circular failure with constant radial growth rate, the resulting rate based on an area of $862-\mathrm{in}^{2}$ and destruction time of 110.250-seconds (51.462-seconds after first flame $)^{2}$ would be approximately $0.32-\mathrm{in} / \mathrm{sec}$. Results of the parametric model using this erosion rate are shown in Fig. 6. The Circular assumption does not initially decay pressure as quickly as the measured data indicates. Assuming an erosion rate of 0.32 -in/sec causes the predicted pressure in excess of the measured data after less than ten seconds.

To best match the measured data, a constant area growth rate of $3-\mathrm{in}^{2} / \mathrm{sec}$, as shown in Fig. 7, was determined. This growth rate does not lead to the size of the apparent failure at the time of motor destruction. To achieve the observed failure size in the time from first flame to destruction, with a constant area growth rate would require a rate of $16.75-\mathrm{in}^{2} / \mathrm{sec}$. These differences highlight the fact that the failure itself was not so easily modeled. Reality proves that the growth rate of the failure may not be linear, or even continuous. In addition, events after the loss of the data stream may have caused additional damage.

Figure 7 STS-51L Modeled with failure growth rate of $3-\mathrm{in}^{2} / \mathrm{sec}$ 


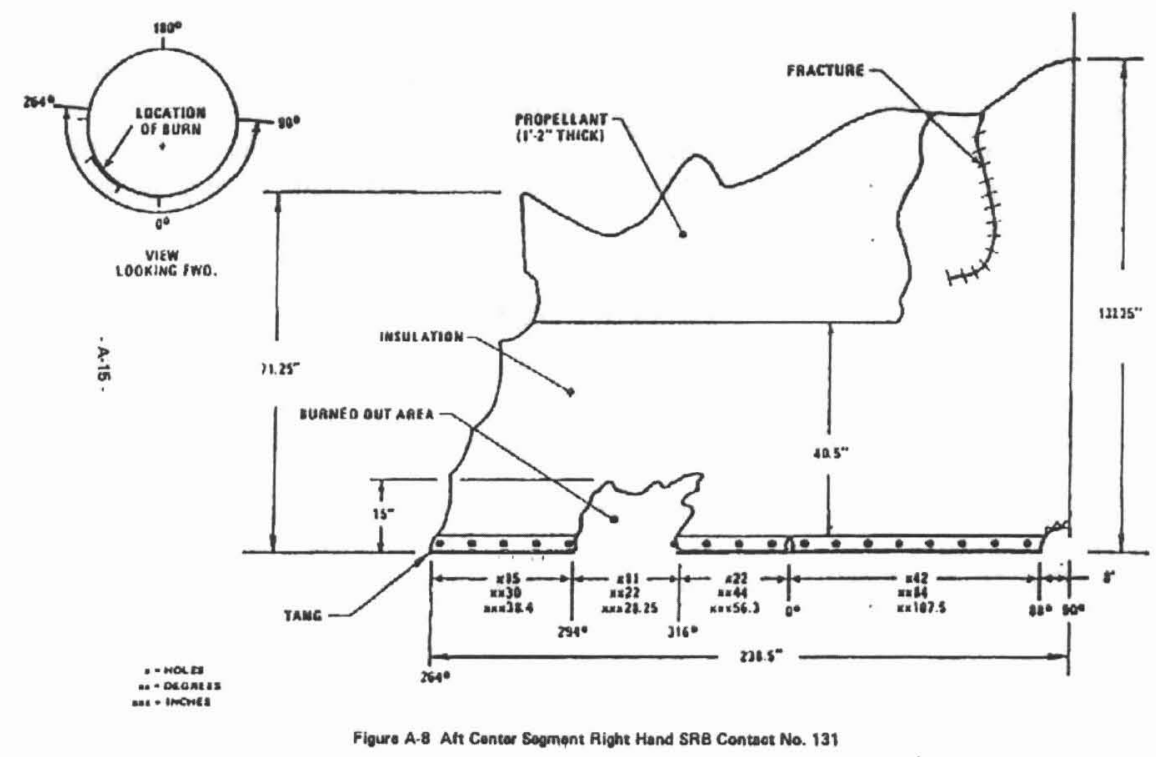

Figure 8: Contact 131, RH Aft Center Segment Aft Cylinder ${ }^{4}$

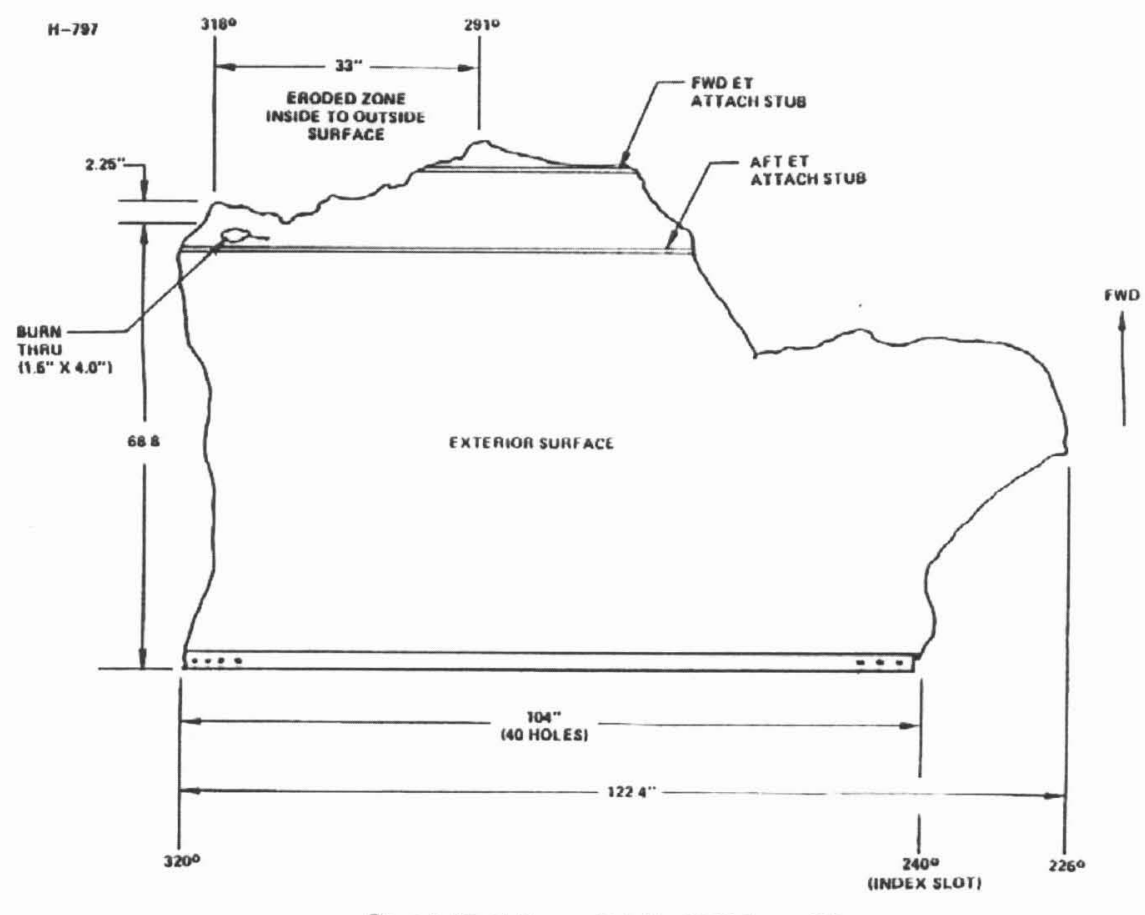

Figure 9: Contact 712, Aft Segment Right Hand Booster Forward Cylinder ${ }^{4}$ 


\section{CONCLUSIONS}

The scaling approach can be used to model motor performance for a given failure. Using fundamental engineering equations, a robust algorithm has been derived for modeling the performance of a booster with a breach in the pressure vessel. For future analyses, simple modifications can be made to this model. Such changes may include modifying the failure growth rate, failure shape, or using the scaling approach to model high rate nozzle throat erosion that would also require modeling the change in expansion ratio. Evaluation of the Challenger data shows that the model is reasonable and adaptable. This tool provides the ability to model abnormal motor performance for use in evaluating contingency scenarios.

\section{REFERENCES}

\footnotetext{
${ }^{1}$ Humble, R. W., Gary N. H., and Wiley J. L., Space Propulsion Analysis and Design, $1^{\text {st }}$ ed.-Revised, The McGraw-Hill Companies, Inc., New York, 1995, Chap. 3.3.

2 "Report of the PRESIDENTIAL COMMISSION on the Space Shuttle Challenger Accident," Vol. 1, Chap. 3, Washington D.C., 1986.

3 "Report of the PRESIDENTIAL COMMISSION on the Space Shuttle Challenger Accident," Vol. 3, Appendix O, Enclosure 9, Washington D.C., 1986.

4 "Report of the PRESIDENTIAL COMMISSION on the Space Shuttle Challenger Accident," Vol. 2, Appendix L, Appendix A, Washington D.C., 1986.
} 